\title{
Fragmented QRS Dynamics Towards Electrical Storm in ICD Patients
}

\author{
Amalia Villa $^{1}$, Sebastian Ingelaere ${ }^{2}$, Sabine Van Huffel $^{1}$, Rik Willems ${ }^{2}$, Carolina Varon ${ }^{3}$ \\ ${ }^{1}$ KU Leuven, Department of Electrical Engineering (ESAT), STADIUS, Leuven, Belgium \\ ${ }^{2}$ Division of Experimental Cardiology, Department of Cardiovascular Diseases, KU Leuven, Belgium \\ ${ }^{3}$ Circuits and Systems (CAS) group, Delft University of Technology, The Netherlands
}

\begin{abstract}
Electrical storm (ES) in ICD patients, defined as 3 or more appropriate ICD interventions within a time span of 24 hours, is a medical emergency associated with adverse outcome. However, it is debated if ES is only a marker of progressive near end-stage cardiac disease or an arrhythmogenic entity on its own. Better understanding and prediction are necessary to manage the burden of ES.

The goal of this study is to explore the relation between the presence of fragmented $Q R S(f Q R S)$ and the manifestation of electrical storm in patients with an ICD for ischemic heart disease. A balanced dataset of 100 patients was considered for this study, where 50 patients with ischemic heart disease and dilated cardiomyopathy present ES. 12-lead ECG signals were analyzed from 3 years before until the moment of ES, divided in 4 visits. The fQRS level in the 12-lead ECG data recorded in each visit was automatically quantified with a score between 0 and 1 for each lead. A Friedman test between the first and last visit for each of the groups showed a significant increase in the average level of fragmentation for the patients presenting ES, absent in the control group. This suggests that there is a trend towards deterioration in $f Q R S$ for patients manifesting ES with an ICD for ischemic heart disease.
\end{abstract}

\section{Introduction}

Electrical storm (ES) in ICD patients is defined as 3 or more appropriate ICD interventions within a time span of 24 hours. This medical emergency generally leads to adverse outcome [1]. ES is a difficult to predict dramatic condition with increasing prevalence [2]. Current research still debates if ES is only a marker of progressive near endstage cardiac disease or an arrhythmogenic entity on its own. The underlying pathophysiological mechanisms are not yet fully understood and require further investigation. Furthermore, the development of better predictive models is necessary to reduce its incidence and burden.

Given the fact that arrhythmia is the presenting clinical picture in ES, current tools for arrhythmia prediction can be taken into account to develop a better model. One of the most known markers of arrhythmia is the presence and level of fragmentation in the QRS complexes [3].

The aim of this work is to explore the relation between QRS fragmentation and the manifestation of electrical storm in patients with an ICD. In order to automatically quantify the level of fragmentation in ECG signals, the algorithm proposed in [4] is used. Certain modifications were added to the original algorithm to deal with noisy signals. The fQRS scores of each lead are compared between a group of patients presenting ES and a control group. Additionally, the evolution of the scores over time is studied for each group.

\section{Methodology}

From the UZ Leuven hospital ICD registry, 50 patients (94\% male, $63 \pm 10$ years, $37.3 \pm 14.3 \%$ LVEF at implant) with ischemic cardiomyopathy presenting with ES were identified. Baseline demographics and raw 12-lead ECG data were collected for these patients from implant until storm. As control subjects, 50 patients (94\% male, $62 \pm 12$ years, $34.2 \pm 12.2 \%$ LVEF) from the same registry were included.

In order to make the groups comparable, 4 visits were considered for each ES patient: (1) from 36 to 18 months pre-ES, (2) from 18 to 6 months pre-ES, (3) from 6 months before to days before the storm and (4) from 0 to 48 hours after the storm. 4 visits similarly spaced in time were considered for the control group. Each of these visits consisted of 10 seconds of 12-lead ECG recordings, sampled at 250, 500 or $1000 \mathrm{~Hz}$.

\subsection{Preprocessing and Segmentation}

Baseline wander and high frequency noise were removed from each lead of the ECG using a high-pass and a low-pass fourth-order Butterworth filter, with 0.5 and $70 \mathrm{~Hz}$ of cut-off frequencies, respectively. Following [4], these filters were applied bidirectionally to obtain zerophase distortion, and each lead was standardized using z- 
score to remove amplitude differences between recordings. Recordings presenting ventricular pacing or arrhythmia, such as atrial fibrillation or ventricular tachycardia, were manually removed from the study, since the algorithm used to quantify fragmentation did not consider such segments in its training [4]. Table 1 summarizes the amount of records available for each visit after the removal of recordings presenting arrhythmia.

Table 1. Number of subjects present in each visit

\begin{tabular}{lrrrrr} 
& \multicolumn{3}{c}{ Months pre-ES } & \multicolumn{2}{c}{ Time post-ES } \\
& 36 to 18 & 18 to 6 & 6 to 0 & 0 to $48 \mathrm{~h}$ & \\
\hline ES Patients & 28 & 34 & 36 & & 28 \\
Controls & 30 & 36 & 23 & & 30 \\
\hline
\end{tabular}

The segmentation of the heartbeats was done semiautomatically to exclude the influence of noisy fluctuations around the QRS. The QRS complexes were automatically detected using the R-DECO software [5]. Visual inspection was further performed to remove irregular heart-beats and noisy segments. These corrections were done for lead II. The fiducial points in this lead were automatically translated to the rest of the leads finding maxima in a fixed-size window around the R-peaks in the absolute signal. Once the R-peaks were detected, the QRS complexes were segmented using a symmetric window of length $\sqrt{R R}$ around the R-peak, as suggested in [6].

The last stage of preprocessing consisted of automatically selecting the usable heartbeats in each 10-second segment for each lead based on cross-correlation. Since only lead II was visually inspected, the QRS complexes in other leads might still present artifacts or noise. Therefore, a stage of noise removal was applied to keep only the cleanest heartbeats for each lead. In this stage, each segmented QRS complex is compared with the rest of the heartbeats present in that record by means of normalized cross-correlation. Only those heartbeats with a correlation above a quality limit were selected. By visual inspection, this limit was set to 0.7. If only less than half of the heartbeats were kept for the analysis, the segment was directly rejected. This allows to remove extra noise that could lead to errors in the fQRS quantification.

\subsection{Feature extraction}

The level of QRS fragmentation of each segment was automatically quantified using the algorithm in [4]. This model derives 10 features from the ECG signal using Variational Mode Decomposition (VMD) and Phase-Rectified Signal Averaging (PRSA).

\section{VMD and ECG features}

VMD is a signal analysis technique that allows to adaptively decompose a signal into its principal modes. The criteria to select these modes $u_{k}$ is two-folded: they must be able to optimally reconstruct the original signal, and they must be band-limited around a central frequency $\omega_{k}$ that is automatically selected [7]. This method has been applied to ECG signals for heartbeat classification [8] and QRS segmentation [4]. One of the main strengths of VMD in ECG analysis is that it allows to identify the frequencies corresponding to the characteristic waves, i.e. the P,Q,R,S and $\mathrm{T}$ waves.

Using the parameters defined in [4] to apply VMD to ECG signals, the number of modes $k$ was set to 5. Since fragmentation presents as additional high frequency components in the ECG signal, the features derived from VMD for $\mathrm{fQRS}$ quantification are obtained from the modes corresponding to the highest frequencies of the signal, i.e. $u_{3}$, $u_{4}$ and $u_{5}$. The features defined to capture fragmentation are the number of local optima per segmented QRS and the central frequencies $\omega_{k}$ of the 3 highest modes, leading to 6 VMD-based features.

An additional feature was directly derived from the ECG, corresponding to the number of local peaks in each QRS.

\section{PRSA features}

The additional 3 features were obtained from PRSA. This technique is used to detect quasi-periodic oscillations in non-stationary signals. While it had been used to evaluate the adaptation capacity of the heart by applying it to the tachogram [9], the work in [10] applied it directly to the ECG to characterize fragmentation. PRSA consists of 3 steps. Firstly, a set of anchor points is selected, in the increasing and the decreasing part of the QRS complex. A modification was here applied to the original method for feature extraction, to ensure the reduction of the influence of oscilations potentially adjacent to the QRS, often related to noise. In order to reject the increases and decreases potentially present in the signal at the sides of the $\mathrm{QRS}$, a mask was applied to the QRS. This mask has 2 different regions corresponding to the center and the sides of the QRS, dividing the length of the QRS as 20-60-20. A different search method is applied to each of the stages of the mask. In the center of the mask, the selection of anchor points is made using the derivative of the signal, as proposed in [4]. This allows to sensitively detect segments of increase or decrease of the signal, which is required to find the short high-frequency fluctuations corresponding to fragmented QRS. In the sides of the segmented QRS, a less sensitive technique is applied to select the anchor points. This is done by constructing a derivative based on the average of the previous $s$ samples to the anchor point, setting $s$ to $5 \%$ of the complete length of the segment.

The second stage consists of applying a window around the anchor point of length $2 L$, with $L=20 \mathrm{~ms}$. Finally, 
all segments are averaged to obtain the PRSA curve, from which the 3 features are derived. It has been shown in [10] and [4] that the averaged segment of fragmented QRS examples is flatter than that of healthy QRS segments. Thus, the 3 features derived from the average segment are the slope of its linear fit, the magnitude of the crossing of the linear fit with the $y$ axis, and the mean derivative of the obtained segment.

\subsection{Automatic quantification of QRS frag- mentation}

Once the 10 features are extracted, they are fed to the model described in [4], which assigns a score between 0 and 1 to each lead of each ECG signal. This model was built using a Support Vector Machines classifier (SVM), which was trained with 10 seconds ECG signals from UZ Leuven, which were annotated by 5 experienced cardiologists as 0 or 1 , for the absence or presence of fragmentation. The training samples were finally labeled from 1 to 5 corresponding to the addition of the scores given by the cardiologists. This resulted in higher scores for those QRS which were clearly fragmented, and lower scores for non or more doubtfully fragmented segments. Platt scaling was used to transform the binary output of the classifier into a continuous scale from 0 to 1 , using logistic regression.

For the data presented in this study, the classifier was applied to each of the visits available for each patient, leading to a set of 12 scores per visit per patient. A set of 4 global scores were derived for each of these visits: the average of the 12 leads, the median, the standard deviation and the maximum score obtained.

\subsection{Statistical Analysis}

Two hypotheses were formulated to see the relation between fQRS scores and the manifestation of ES. For the first hypothesis, a difference in the level of fragmentation between patients presenting ES and control patients was expected. This was evaluated using the Wilcoxon-Mann-Whitney test for each of the 3 global scores derived from the data in the last visit of the patients, corresponding to the moment after storm. In particular, the levels of fragmentation were hypothesized to be higher for the ES patients than for the controls.

The second hypothesis proposed aimed to find trends over time for each of the groups. By comparing the global scores between the first and consecutive visits, different trends were expected to be found for each of the groups. This test was evaluated using the Friedman test for paired data. Therefore, only the patients who had records for both the first and the last visit were included in this test.

\section{Results and discussion}

Figure 1 shows the boxplots of both groups in the last visit for the different global scores considered. ES patients are referred to as ES and control patients as controls. A slight difference can be observed between both groups, generally indicating a higher global fQRS score for ES than controls, together with a higher standard deviation. This is aligned with the first hypothesis. Nevertheless, the Wilcoxon test did not show significant differences between both groups in any of the considered global metrics.

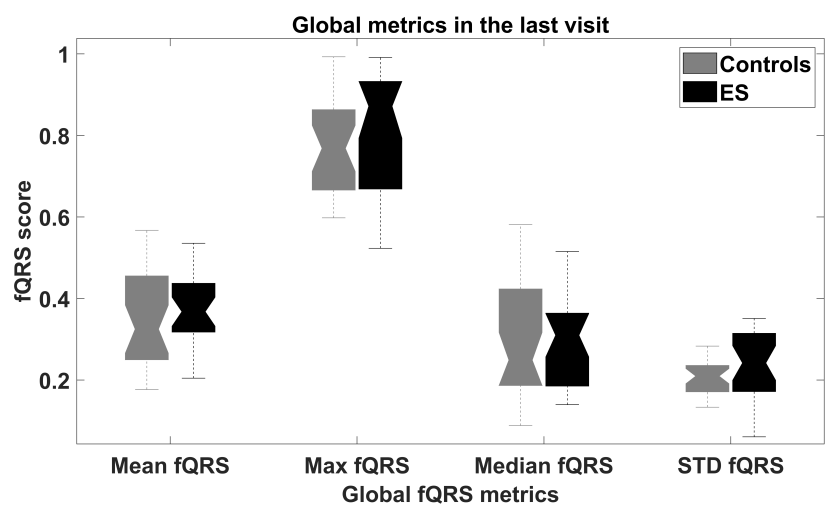

Figure 1. Global fragmentation scores for the last visit of ES patients and control patients.

Figure 2 shows the boxplots of both groups from the first to the last visit around ES for the average fQRS score. An increasing trend can be observed in the ES group, which is not visible in the controls. The highest scores are obtained in the third visit, around 6 months before the storm, and these values remain for the last visit.

In order to confirm this observation, a Friedman test was performed. This was done to compare the average score of the first and the last visit, for each of the groups. Since this is a test for paired data, only the patients who had records for both visits were included in this test. This lead to 28 and $30 \mathrm{ES}$ and control patients, respectively. While the control patients obtained a $p$-value of 0.67 , the ES patients show significant differences with a $p$-value $<0.05$.

These results suggest that there is a trend towards deterioration in those ICD patients that manifest electrical storm, which is not present in the control patients. This is reflected in a higher average fragmentation score of the leads. This is aligned with the literature, that suggests fQRS as an indicator of potential arrhythmia [3], which could eventually become ES. This confirms the need to closely monitor the level of QRS fragmentation in ES patients.

Nevertheless, these are preliminary results which require further study, since this work presents some limitations. First of all, the use of an automatic tool for fQRS 


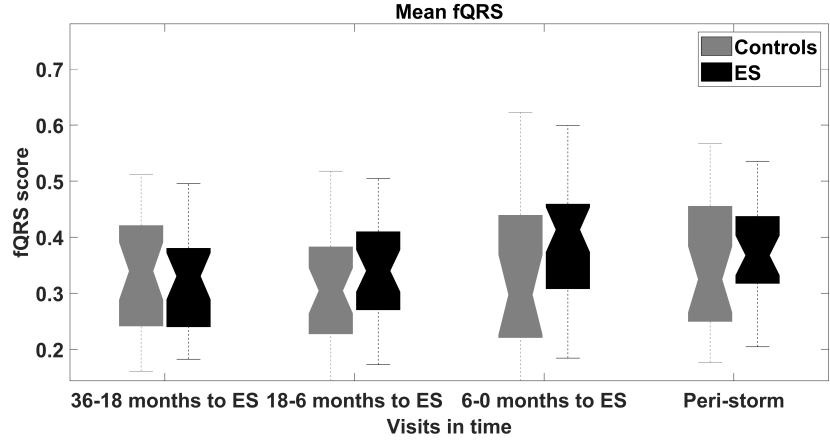

Figure 2. Average fragmentation score along the visits, from 3 years before to the time around electrical storm.

quantification may lead to inaccurate results. While fQRS quantification is a challenging task even for experienced cardiologists, the use of an algorithm for this aim can introduce additional errors. One of the main sources of error is the influence of noisy QRS complexes. Since the complete dataset was not visually inspected, i.e. not all the leads were supervised, noisy QRS may have been considered in the analysis, leading to biased or erroneous results. Future work will aim to tackle this problem.

Additionally, the algorithm used for fQRS quantification provides an independent score for each lead, while the statistical analysis requires a global score that allows to compare all patients. The definition of this global score requires further investigation, as well as the relation of new regional scores with the type implanted ICD.

\section{Conclusion}

A study exploring the relation between QRS fragmentation and the manifestation of ES in patients with ischemic heart disease and ICD is presented. The dynamics of QRS fragmentation in 50 patients with a history of ES were compared to 50 control patients without it.

The quantification of fQRS was done automatically using a state-of-the-art algorithm, and the results were evaluated over time. A trend towards fQRS deterioration in patients presenting ES was discovered from around 3 years before the event. While further potential improvements are required for the fQRS algorithm, as well as the definition of new global scores for 12-lead ECG, this work suggests the relevance of fQRS in the presented group of patients as a potential biomarker of the risk of ventricular storm.

\section{Acknowledgements}

Research funded by Agentschap Innoveren en Ondernemen (VLAIO) 150466: OSA+. KU Leuven Stadius acknowledges the financial support of imec. This research received funding from the Flemish Government (AI Research Program). SVH, AV and CV affiliated to Leuven.AI - KU Leuven institute for AI, B-3000, Leuven, Belgium. $\mathrm{R}$. Willems is supported as postdoctoral clinical researcher by the Fund for Scientific Research Flanders.

\section{References}

[1] Eifling M, et al. The evaluation and management of electrical storm. Texas Heart Institute Journal 2011;38(2):111.

[2] Hohnloser $\mathrm{SH}$, et al. Electrical storm in patients with an implantable defibrillator: incidence, features, and preventive therapy: insights from a randomized trial. European heart journal 2006;27(24):3027-3032.

[3] Das MK, et al. Fragmented QRS on 12-lead electrocardiogram predicts arrhythmic events in patients with ischemic and nonischemic cardiomyopathy. Heart rhythm 2010; $7(1): 74-80$.

[4] Goovaerts $\mathrm{G}$, et al. A machine-learning approach for detection and quantification of QRS fragmentation. IEEE Journal of Biomedical and Health Informatics sep 2019; 23(5):1980-1989. ISSN 21682208.

[5] Moeyersons J, et al. R-DECO: An open-source matlab based graphical user interface for the detection and correction of R-peaks. PeerJ Computer Science oct 2019; 2019(10):e226. ISSN 23765992.

[6] Clifford G, et al. Advanced methods and tools for ECG data analysis. Artech house Boston, 2006.

[7] Dragomiretskiy K, Zosso D. Variational mode decomposition. IEEE transactions on signal processing 2013; 62(3):531-544

[8] Villa A, et al. Variational mode decomposition features for heartbeat classification. In 2018 Computing in Cardiology Conference (CinC), volume 45. IEEE, 2018; 1-4.

[9] Schneider R, et al. Phase-rectified signal averaging for the detection of quasi-periodicities in electrocardiogram. In IFMBE Proceedings, volume 16. Springer Verlag. ISBN 9783540730439. ISSN 14339277, 2007; 38-41.

[10] Goovaerts G, et al. Phase-rectified signal averaging for automatic detection of QRS fragmentation. In 2016 Computing in Cardiology Conference (CinC). 2016; 637-640.

Address for correspondence:

Amalia Villa

Department of Electrical Engineering (ESAT),

STADIUS, KU Leuven, Belgium

amalia.villagomez@kuleuven.be 